\title{
Evidence for Nonlinear Development of MHD scale Intermittency in the Inner Heliosphere
}

\author{
A. Greco ${ }^{1}$, W. H. Matthaeus ${ }^{2}$, R. D'Amicis ${ }^{3}$, S. Servidio ${ }^{1}$, P. Dmitruk ${ }^{4}$
}

\begin{abstract}
The formation of coherent structures in turbulence is a signature of a developing cascade and therefore might be observable by analysis of inner heliospheric solar wind turbulence. To test this idea, data from the Helios 2 mission, for six streams of solar wind at different heliocentric distances and of different velocities, is subject to statistical analysis using the Partial Variance of Increments (PVI) approach. We see a clear increase of PVI distribution function vs solar wind age for higher PVI cutoff, indicating development of non Gaussian coherent structures. The plausibility of this interpretation is confirmed by a similar behaviour observed in two dimensional magnetohydrodynamics simulation data at corresponding dimensionless nonlinear times.
\end{abstract}

Subject headings: Magnetohydrodynamics (MHD)- solar wind - Turbulence

\section{Introduction}

The formation of coherent structures that are characterized by non Gaussian statistics at small scales is a well known feature of fully developed turbulence, in both hydrodynamics (Sreenivasan \& Antonia 1997) and magnetohydrodynamics (MHD) (Biskamp \& Müller 2000). Simulations indicate that these structures form rapidly in the development of a cascade, and are driven by ideal nonlinear dynamics (Frisch et al. 1983; Wan et al. 2009). Here we explore the dynamical development of solar wind turbulence observed in the inner heliosphere to understand whether statistical signatures of the development of intermittency are present.

\footnotetext{
${ }^{1}$ Dipartimento di Fisica, Università della Calabria, Rende, Italia

${ }^{2}$ Bartol Research Institute, Department of Physics and Astronomy, University of Delaware, Delaware, USA

${ }^{3}$ INAF - Istituto di Fisica dello Spazio Interplanetario, Rome, Italia

${ }^{4}$ Instituto de Astronomía y Física del Espacio (CONICET-UBA) and Departamento de Física (FCENUBA), Buenos Aires, Argentina
} 
To this end we will examine the radial development of statistical measures of non Gaussianity using Helios 2 data, and compare the results with MHD simulation data designed to evolve over a number of nonlinear timescales that is similar to estimates of the nonlinear aging in the solar wind within the observed range of distances. Indeed the evidence will suggest that there is comparable development of intermittency in the two cases.

The evolution of solar wind fluctuations in the inner heliosphere (within $1 \mathrm{AU}=$ Astronomical Unit) is well documented (Bavassano et al. 1982; Tu et al. 1984; Bruno et al. 1985; Roberts et al. 1987; Tu 1988; Tu \& Marsch 1995; Bruno \& Carbone 2005) but due to the complexity of the plasma physics involved and the variability of the solar sources, this remains an active area of research. Inner heliospheric MHD scale velocity and magnetic field fluctuations are broadband and at periods of less than a few hours are typically correlated with one another in the sense of outward traveling Alfven waves (Belcher \& Davis 1971). A range of power law spectra is observed, with the low frequency cutoff migrating towards lower frequencies with increasing distance (Bavassano et al. 1982). A familiar interpretation is that the presence of the observed power law "Kolmogorov" spectra is indicative of dynamically generated local correlations and an active in situ turbulence cascade (Tu et al. 1984; Tu 1988). This conclusion is favored by the migration of the increase in scale of the break-point, interpreted as further evidence of in situ nonlinear evolution ( $\mathrm{Tu}$ et al. 1984; Matthaeus \& Goldstein 1986). In addition the correlation of velocity and magnetic field correlations ("Alfvenicity") decreases on average with increasing heliocentric distance (Bruno et al. 1985; Roberts et al. 1987). The decrease in Alfvenicity can be similarly interpreted as due to driving of the cascade by large scale shear, an effect also observed in turbulence simulations (Roberts et al. 1992). A two-component incompressible fluctuation model also explain the observed decrease in Alfvenicity and the ratio between the energies of velocity and magnetic fluctuations ( Tu \& Marsch 1993). Other properties, such as the degree of non-equipartition of velocity and magnetic field turbulence energy, also evolve with increasing distance towards a state that appears to be more consistent with fully developed turbulence (Marsch \& Tu 1990). Concomitantly there is also an observed heating (Coleman 1968; Tu 1988; Breech et al. 2008) that apparently is also consistent with an explanation due to a turbulence cascade that leads to enhanced dissipation.

The various lines of reasoning alluded to above point to the presence of evolving MHD scale turbulence in the inner heliosphere. The development of the spectral break between $f^{-1}$ and $f^{-5 / 3}$ frequency regions, and the evolution of the Alfven ratio and the cross helicity, are some of the features that suggest the early stages of evolution of turbulence from an initial highly Alfvenic state close to the sun.

There are, however, some aspects of the evolution of interplanetary plasma fluctuations 
that remain poorly understood, or are controversial.

A feature that is often studied but incompletely understood is the intermittency or small scale non Gausssianity of turbulent fluctuations. It is well known from studies of hydrodynamics and MHD that strong "fully developed" turbulence is characterized by the appearance of small scale coherent structures that are responsible for the observed intermittency of the small scale fluctuations (Sreenivasan \& Antonia 1997; Anselmet et al. 1984). These structures play a special and important role in turbulence theory because they are sites of enhanced dissipation, and therefore their non Gaussian statistics are related to the intermittent nonuniform distribution of the dissipation. The conjectured statistical relationship between the statistics of dissipation and the statistics of small scale fluctuations is known as the Refined Similarity Hypothesis, which although unproven, provides theoretical underpinning to studies of multifractals and other scaling behavior. Such non Gaussian distributions and characteristic scalings are also observed in MHD and in the solar wind (Biskamp \& Müller 2000; Burlaga 1991; Marsch \& Tu 1994; Carbone 1994; Horbury \& Balogh 1997; Sorriso-Valvo et al. 1999; Bruno \& Carbone 2005). The observed scalings are faithful enough to the standard expectations to bolster the view that the solar wind is an intermittent turbulent medium, but is it an active turbulent medium?

One way to address this issue is to try to answer the following question: Do the statistical signatures of coherent structures evolve with heliocentric distance in the inner heliosphere, in analogy to the evolution of the break-point, the Alfvenicity and the Alfven ratio? Better understanding of this issue may provide insight into the origin of solar wind fluctuations and the relationship between the coronal and interplanetary plasmas, as we will discuss further below. This is the question that we address in the present paper.

Before proceeding with our analysis it is important to recognize a related study by Bruno et al. (2003), that investigated the radial evolution of magnetic field intermittency in the inner heliosphere using Helios data. This has much in common with the study reported below, but both the analysis approach and the theoretical perspective differ. Bruno et al. (2003) examine the flatness, i.e. the normalized fourth order moment, of the probability distribution functions. They found a different behavior for slow and fast wind intermittency. Even though slow wind is more intermittent than fast wind, it does not show a strong radial dependence, while fast wind intermittency clearly increases with heliocentric distance. The same authors have interpreted this trend considering the different role played by coherent advected structures and by propagating stochastic Alfvenic fluctuations in fast and slow wind at different heliocentric distances. As a matter of fact, in fast wind the Alfvenic contribution, which tends to decrease intermittency because of its stochastic nature, is depleted as the wind expands. (We recall that this depletion of Alfvenicity has been attributed to turbulence 
(Roberts et al. 1992) as discussed above.) On the other hand, advected structures tend to increase intermittency because of their coherent nature while their contribution becomes more important with increasing heliocentric distance. Slow wind does not show the same behavior since for this type of wind, Alfvenic fluctuations have a less dominant role.

The present results are consistent with Bruno et al. (2003). However the interpretation will be somewhat different because we will attribute the differing behavior in various samples of solar wind turbulence to the role played by the turbulence age (Matthaeus et al. 1998), measured in characteristic nonlinear times. We discuss this further at the end of the paper. We will also argue that both our results and those of Bruno et al. (2003) strongly favor the interpretation that the solar wind MHD scale cascade is active and not passive as sometimes suggested (Borovsky 2008). The present results also raise some interesting questions regarding the initial-boundary data for solar wind fluctuations.

Our approach in demonstrating these conclusions will consist of an observational component in which we make use of a variation of the methods based on the moments of the Probability Distribution Functions (PDFs) of magnetic increments. We make use of the partial variance of increments method (PVI) (Greco et al. 2008). Instead of employing this diagnostic only to identify events above a certain threshold, we introduce an alternative strategy based on the computation of the cumulative probability of finding the PVI statistics above a specified threshold. This can be interpreted as the fractional volume occupied by quasi-discontinuous coherent structures. The higher the cumulative probability, the greater is the density of strong discontinuities present. The second novel feature of this study is that we also employ the same methodology to the analysis of MHD turbulence simulation data. By comparing the evolution of cumulative PVI event probability in observations and in simulations at similar estimated nonlinear times, we can evaluate the possibility that the evolution of the intermittency is associated with aging of the turbulence.

Before presenting the main results in Section 3, in the next section will describe the data analysis procedure and the MHD simulation. Section 4 will summarize the findings and discuss implications for understanding the dynamics of solar wind turbulence in the inner heliosphere.

\section{Magnetic field data}

The only available dataset that can address the questions raised above is data from the Helios mission. The present data analysis is based on $6 \mathrm{~s}$ averages of magnetic field recorded by the Rome/GSFC magnetometer on Helios 2 during its primary mission to the sun in 1976. 
The dataset consists of fast and slow solar wind time periods at different heliocentric distances. In particular, the fast solar wind intervals correspond to the same corotating stream at three different heliocentric distances, during three successive solar rotations (Bavassano et al. 1982). For each stream we selected a time interval within the trailing edge and a slow wind interval ahead of it. The length of each period is about 2 days. Gaps found within were linearly interpolated. However, gaps do not exceed $10 \%$ of each period. The selected intervals, the relative average heliocentric distance and the average solar wind speed are reported in Table 1.

Bavassano et al. (1982) computed the power spectra of the three fast streams we are studying in this paper, showing that they are characterized by two distinct spectral slopes: an approximately -1 range in the low frequencies and an approximately Kolmogorov-like $-5 / 3$ spectrum at higher frequencies. The two regimes are separated by a frequency "break point" that moves to lower frequencies as the wind expands (Bavassano \& Smith 1986; Klein et al. 1992; Bruno \& Carbone 2005). The shift to lower frequencies, during the wind expansion, is consistent with the growth of the correlation length observed in the inner heliosphere (Bruno \& Dobrowolny 1986) and the corresponding contraction of the $1 / f$ range due to dynamical generation of correlations (Matthaeus \& Goldstein 1986). Slow wind power spectra, on the contrary, are characterized by a single power law with a Kolmogorov-like slope covering the whole frequency range at the three heliocentric distances considered here.

The behaviour of the power spectra, correlation lengths and intermittency of the magnetic field fluctuations have been confirmed by a recent study by D'Amicis et al. (2010) on the radial evolution of turbulence of the same fast wind plasma sample observed at two heliocentric distances during a radial alignment occurred between Earth and Ulysses at the end of August 2007. A familiar interpretation (e.g., Matthaeus \& Goldstein (1986)) of this evolution is the growth of the correlation scale due to dynamical involvement of larger structures that is expected in evolving turbulence. Conversely, the very low frequency $1 / f$ spectrum, in this interpretation, is viewed as generated in the subAlfvenic corona and thus representing the time dependence of the sources of the solar wind.

\section{Statistical Analysis}

To identify coherent structures through rapid changes in the magnetic field components, we examine the increments

$$
\Delta \mathbf{b}(s, \Delta s)=\mathbf{b}(s+\Delta s)-\mathbf{b}(s)
$$


where $\boldsymbol{b}$ is the magnetic field vector. The $1 \mathrm{D}$ coordinate along the spacecraft trajectory is $s$, which is a time coordinate. Using the Taylor hypothesis, the time signal is converted to a spatial signal using the average velocity of the flow. The spatial separation or lag is $\Delta s$.

For this analysis we choose a time lag of $6 \mathrm{sec}$ for the fast streams and of $12 \mathrm{sec}$ for the slow streams. These time lags are sufficiently small (consistently with the resolution of the samples) to sample the very intermittent structures (almost at the end of the inertial range).

Employing only the sequence of magnetic increments, we compute the normalized magnitude

$$
P V I=\frac{|\Delta \mathbf{b}(s, \Delta s)|}{\sqrt{\left\langle|\Delta \boldsymbol{b}(s, \Delta s)|^{2}\right\rangle}},
$$

where $\langle\bullet\rangle$ denotes a spatial average over the entire dataset (2 days), and $\Delta s$ is the spatial lag in Eq. (1). The square of the above quantity has been called the Partial Variance of Increments (PVI) (Greco et al. 2008) and the method abbreviated as the PVI method. The performance of the PVI to identify discontinuities has been shown to be comparable to standard methods (e.g., Tsurutani \& Smith (1979)) in both MHD simulations and solar wind observations (Greco et al. 2008, 2009a). Note that by construction $\left\langle P V I^{2}\right\rangle=1$, while $\left\langle P V I^{4}\right\rangle$ is related to the kurtosis of the magnetic field component increments. Furthermore, the powers of $\langle P V I\rangle$ scale in a manner that are connected with familiar diagnostics of intermittency (Horbury et al. 1997). An example of the PVI time series computed for 4 of the six streams is shown Figure 1, where the sampling time $t$ is normalized to the correlation time computed for each stream (see section 4 for a description of the computation of the correlation times and Table 2 for their typical values). Here, we analyze the statistical distribution of Eq. (2), computing the probability density functions (PDFs) for all six streams, as displayed in Figure 2. All the PVI distributions have a probability density that increases at smaller values towards an enhancement for the intermediate value bins, and then drop for the largest value bins.

There are several features to be noted in the distributions of PVI. First, the slow streams have a larger probability for high PVI values than do the fast streams. This means, in the usual interpretation, that the slow wind is more intermittent (Bruno et al. 2003), in that it contains a greater concentration of coherent structures. Moreover it is apparent, by examination of the tails of the distributions in the inset of Figure 2, that slow wind does not show a clear radial dependence, while fast wind does. This confirms the results of Bruno et al. (2003) and also Tu et al. (1996); however in the following section we develop an alternative interpretation.

To proceed with the analysis we further describe the nature and occurrence of the PVI "events" that make up the tails of the PVI distributions. In particular, imposing a threshold 
$\theta$ on Eq. (2), a collection of these events, which are in effect, discontinuities, or coherent structures, can be identified along the path in $s$. We select portions of the trajectory in which the condition

$$
P V I>\theta
$$

is satisfied. To better understand the physical meaning of the threshold $\theta$, we recall that the probability distribution of the PVI statistic derived from a non Gaussian turbulent signal is empirically found to strongly deviate from the PDF of PVI computed from a Gaussian signal, for values of PVI greater than about 3 (Greco et al. 2008, 2009a). As PVI increases to values of 4 or more, the recorded "events" are extremely likely to be associated with coherent structures and therefore inconsistent with a signal having random phases. Thus, as $\theta$ is increased, stronger and more rare events are identified, associated with highly non Gaussian coherent structures.

A previous analysis of solar wind ACE data (Greco et al. 2009b) has verified that power laws describe the distribution of waiting times (inter-arrival times) (WTs) between PVI events, for periods up to $1000-3000 \mathrm{~s}(\sim 50 \mathrm{~min})$, which is of the order of the correlation time of magnetic fluctuations in the solar wind. The scaling exponent inferred from a linear fit was -1.23 . Here, we computed the distribution of WTs for the slow stream at 0.9 AU. As in previous works, the distribution shown in Figure 3 is well fit by a power law for periods up to $\sim 1000-3000 \mathrm{~s}$. Here, the scaling exponent inferred from a linear fit, is -2.3 .

The difference between the values of the two power law indexes maybe due to the fact that for Helios we considered slow wind at $0.9 \mathrm{AU}$ and time separation of 12 sec. For ACE it was "mixed" wind at $1 \mathrm{AU}$ and the time separation was $4 \mathrm{~min}$. Indeed when we considered different time separations for PVI (e.g. up to $10 \mathrm{~min}$ ), we got shallower indexes for PDF(WTs) (not shown). This is very interesting, but indeed represents a separate study.

Previously, some authors (Carbone et al. 2003), using the same stream, have shown a very similar power law for events identified with somewhat different methods, extending in the same range of time separations, with a scaling exponent of -2.18 . In that case, the presence of those structures has been evidenced by using a wavelet procedure (Veltri \& Mangeney 1999; Bruno et al. 2001).

\section{Intermittent properties vs the solar wind age}

MHD scale fluctuations have been seen at all distances at which spacecraft have visited. Therefore a reasonable supposition is that these fluctuations at least in part originate lower in the solar atmosphere, entering the super-Alfvenic wind in the trans-Alfvenic critical 
region (probably) near 15-25 $R_{s}$. Along with subsequent expansion and evolution (Tu et al. 1984), the fluctuations are most likely also driven, in the inner heliosphere, by shear at stream interfaces (Roberts et al. 1992; Zank et al. 1996) and by other transients. Transport theories that incorporate these ongoing dynamical effects have been reasonably successful in explaining observations ranging from Helios in the inner heliosphere, to Voyager and Pioneer beyond 50 AU (Tu 1988; Smith et al. 2001; Breech et al. 2008). One of the lessons learned by this kind of studies is that the fast wind "ages" more slowly with heliocentric distance due to smaller convection times (Matthaeus et al. 1998); nevertheless the same basic physics operates in both fast and slow winds, although with apparently differing initial/boundary data and with somewhat different sets of parameters (Matthaeus et al. 2004; Breech et al. 2005, 2008; Ng et al. 2010). Given this background it seems reasonable to examine whether the intermittency properties observed in solar wind turbulence are also developing dynamically, along with energy densities, cross helicities, correlation lengths and other parameters that are apparently developing and aging with intrinsic turbulence and expansion timescales.

In this regard, using the heliocentric distance $R$ and the average bulk speed $U$ shown in the second and third columns of Table 1, we calculate for the six streams analyzed here the estimated convective "age" $t_{\text {conv }}=R / U$. This very elementary solar wind age, in terms of clock time since leaving the sun, is displayed in the fourth column of the same table. We note that the ages differ by up to factor of five, so there is ample possibility that dynamical aging may influence the intermittency properties as seen in the PVI distributions in Figure 2 .

At this point, it is interesting to see if the tails of the PVI distributions, shown in Figure 2 develop systematically with increasing heliocentric distance in the Helios 2 observations. We calculated PVI distribution function integrated over all values $>\theta$, that is the total probability that a signal is observed at any value greater than the cutoff. The time history of this quantity is displayed in Figure 4 , with $\theta=3,4,5,6$.

The plots, especially for higher cutoff, shows a clear trend upwards with age. This may indicate that the turbulence is developing stronger intermittent events as the wind ages while propagating away from the Sun.

\section{Simulation}

The convection time and Alfven crossing times are both reasonable time units for solar wind evolution, although they measure different effects. Still other possibilities exist, such as the "collisional age" that appears to order the development and saturation of kinetic 
effects (Kasper et al. 2008). In spite of their distinct physical origins, these time scales do not evolve very differently within the range of Helios orbits, and so it may be a matter of some subtlety to distinguish their relative prominence. Physical reasoning provides guidance in suggesting for example that evolution of large structures is most sensitive to expansion, while distribution functions have a greater sensitivity to collisional aging. Similarly, it is reasonable to suppose that the turbulence dynamics itself, already known to explain many features of the evolution of the fluctuations at the MHD scales $(<0.01 \mathrm{AU})$, are likely to be responsible for dynamical changes in the degree of intermittency. A straightforward way to examine the plausibility of this is to examine whether turbulence observed in numerical simulations exhibits development of intermittency in a similar way to what is seen in the above solar wind observations.

To this end, we examined data from an MHD turbulence simulation that starts with random phases and therefore initially lacks non Gaussian inertial range coherent structures. Following this evolution, we examined the time history of the same quantities that we have calculated above.

In particular, the simulation solves the $2 \mathrm{D}$ incompressible MHD equations written in terms of the magnetic potential $a(x, y)$ and a single component of vorticity $\omega(x, y)$, with uniform mass density $\rho=1$. The velocity field $\boldsymbol{v}$ is solenoidal, $\nabla \cdot \boldsymbol{v}=0$, the magnetic field is $\boldsymbol{b}=\nabla a \times \hat{\boldsymbol{z}}$, and the the electric current density is $j=-\nabla^{2} a$. The velocity $\boldsymbol{v}=\nabla \phi \times \hat{\boldsymbol{z}}$ can be written in terms of a stream function $\phi$, related to the vorticity by $\omega=-\nabla^{2} \phi$. The equations are written in familiar Alfven units with lengths scaled to $L_{0}$, a typical large scale length. All quantities are in a set of dimensionless units defined by scaling velocities and magnetic fields to the root mean square Alfven speed $C_{A}$, while time is scaled to $L_{0} / C_{A}$. The constant coefficients $R_{\mu}$ and $R_{\nu}$ are reciprocals of kinematic viscosity and resistivity, respectively, and represent magnetic and kinetic Reynolds numbers at scale $L_{0}$ (for more details, see Servidio et al. (2010)). The equations are solved in doubly periodic $(x, y)$ Cartesian geometry in a box of side $2 \pi L_{0}$. In this work we show results from a run with $4096^{2}$ grid points and $R_{\nu}=R_{\mu}=1700$. In the Fourier pseudospectral representation, the fluctuations are confined initially in the shell $4 \leq k \leq 10$, where the wavenumber $k$ is in units of $1 / L_{0}$. Mean values such as energy per unit mass, $E=1 / 2\left\langle|\boldsymbol{v}|^{2}+|\boldsymbol{b}|^{2}\right\rangle$, are expressed in terms of $\langle\bullet\rangle$ which denotes a spatial average. Random phases are employed for the initial Fourier coefficients, which are therefore initially uncorrelated. Initial velocity and magnetic field fluctuations are equipartitioned. With the above parameters, the turbulence correlation length is $\lambda_{C}=1.8 \times 10^{-1}$, and the dissipation length is $\lambda_{d}=4.6 \times 10^{-3}$. 
The peak of the turbulent activity and of the mean square current density is around $t=0.4-0.5$. When the turbulence is fully developed, coherent structures appear. The large scale structures can be identified as magnetic islands that have different size and energy. The dynamics is such that between the islands the perpendicular (out-of-plane) component of the electric current density $j$ becomes very large. These often very thin current structures are the small scale dynamically produced magnetic structures that are characteristic of 2D MHD (these are generally not present in random-phase initial data).

To simulate a data set that is analogous to the spacecraft measurements of the magnetic field along a selected trajectory, we adopt the same technique used by Greco et al. (2008), but here arranged for the 2D case. We interpolate the data along the selected path through the simulation box, and on these data we compute the PVI series from Eq. 2. This permits identification of the current sheets or discontinuities using the PVI method in Eq. 3.

Figure 5 shows PVI series for 4 snapshots of the simulation. Time $t$ is just the "simulation time", computed as $L_{0} / V_{A}$, where $V_{A} \sim 1$ is the Alfven speed ( $B_{r m s}$ is about 1 ). Here, we choose the increment lag to be $\Delta s=0.425 \times 10^{-2} \lambda_{C}$, which is, as in the solar wind case, of the same order of $\lambda_{d}$. From the figure, it is apparent that the PVI time series become more bursty as time passes, up to $t=0.4-0.5$, suggesting the presence of sharp gradients in the magnetic field, and localized coherent structures that represent the spatial intermittency of turbulence (Matthaeus \& Montgomery 1980; Carbone et al. 1990). Since this is a decaying run, the turbulence amplitude decreases about $40 \%$ of what it was initially. Indeed the plot on the bottom shows smaller peaks than the previous times.

The same analysis that was carried out for the solar wind data is also carried out for the simulation data: for several choices of thresholds $P V I>\theta$, the fraction of the PVI distribution falling above the threshold $\theta$ is computed.

\section{Comparison between solar wind and simulation data}

At this point we examine whether turbulence observed in numerical simulations exhibits development of intermittency in a similar way to what is seen in the above solar wind observations. To provide potentially more meaningful comparisons between solar wind and simulation cases, we need to normalized the time of the simulation and the age of solar wind to a suitable time that quantifies the strength of turbulence in both cases. Thus, if we consider the solar wind and an MHD turbulence simulation as different samples of a decaying homogeneous turbulence, we looks at the two systems when the same fraction of initial energy remains. Such turbulence is of equal "age". This convention is familiar in 
numerical simulations when comparing undriven dissipative runs having different Reynolds numbers.

The solar wind age at the position $r$ and relative to a reference position $r_{0}$ has been defined in section 4 as the convective time

$$
t(r)=\int_{r_{0}}^{r} \frac{d r}{U}
$$

where $U$ is the constant convection velocity.

As in Matthaeus et al. (1998) the strength of turbulence can be quantified by the identification of a rate of turbulent evolution. This provides a natural definition of "aging" of the turbulence. A natural clock to measure this "aging" could be the timescale associated with the energy decay in homogeneous MHD turbulence. In the Karman-Taylor approach for modeling the evolution of solar wind turbulence (Matthaeus et al. 1998), the decay timescale becomes the "eddy turnover" time or non-linear time $t_{N L}=\lambda_{C} / Z$, where $\lambda_{C}$ is the correlation scale of the turbulence and $Z^{2}=u^{2}+b^{2}$ ( $u$ and $b$ are the velocity and magnetic fluctuations, respectively), is the Elsasser amplitude associated with the incompressible turbulent energy (in the case of low or zero cross helicity). In this part of the analysis, 81 sec averages of magnetic field and velocity have been used.

By adopting the above model for the energy decay, the solar wind age (4), in term of non-linear times $t_{N L}$ (namely the dimensionless age), is

$$
\tau(r)=\int_{r_{0}}^{r} \frac{d r}{U} \frac{Z(r)}{\lambda_{C}(r)} .
$$

Thus, if the spatial variation of the correlation scale and the turbulent energy density is known, the dynamical age of the turbulence can be directly calculated. Here we employ the Karman-Taylor approach for modeling the evolution of $\lambda_{C}$ and $Z$ (Matthaeus et al. 1998), obtaining the scalings $\left.\lambda_{C}(r) \sim \sqrt{(} r\right)$ and $Z^{2}(r) \sim 1 / r$ (Matthaeus et al. 1998). Then, the corresponding MHD timescale, in case of zero cross helicity, is $t_{N L}(r)=\lambda_{C}(r) / Z(r) \sim r$. However, this simplification is well satisfied beyond few Astronomical Units (AU), but it is not a reasonable simplification at distances less than 1 AU from the sun (Tu et al. 1989). Matthaeus et al. (2004) have shown that for nonzero cross helicity $\sigma_{c}$, along with the equations for the evolution of $\lambda_{C}$ and $Z$, a third simplified equation for $\sigma_{c}$ can be written, which causes a reduction in the effective decay of the above quantities by a factor $f$. In turn, the nonlinear time becomes $t_{N L}(r)=f \lambda_{C}(r) / Z(r)$, where $f$ is a function of $\sigma_{c}$ and it is defined as $\frac{\left(1-\sigma_{c}^{2}\right)^{1 / 2}}{2}\left[\left(1+\sigma_{c}\right)^{1 / 2}+\left(1-\sigma_{c}\right)^{1 / 2}\right]$. The factor $f$ is a decaying function for $\sigma_{c}>0$, and, clearly, if $\sigma_{c}=0 f=1$. 
Therefore, after integrating (5), the age in nonlinear times observed at position $R$ is

$$
\tau(R)=\frac{t_{c o n v}(R)}{t_{N L}(R)} \ln \frac{R}{r_{0}}
$$

where $t_{\text {conv }}(R)=R / U$.

These forms of the correlation scales and turbulent amplitudes, which come from a simple decaying homogeneous turbulence phenomenology (Matthaeus et al. 1998), are supposed to be an approximation to how they behaves if we could follow a stream's evolution, which we cannot do without multiple spacecraft. An alternative phenomenology for turbulent decay (e.g. Dobrowonly et al. (1980)) leads to results which can be readily obtained.

From Helios 2 data, we get the values of $\lambda_{C}$ and $Z$ at each observation point, $R=$ $0.29,0.34,0.65,0.69,0.88,0.9 \mathrm{AU}$, for each stream considered.

For the computation of the correlation scale, we used that associated with the $\mathbf{6}$ s normal component of magnetic fluctuations, because it is less sensitive to sampling of the reversals of the Parker spiral. We approximate $\lambda_{C}$ as the distance over which the autocorrelation function falls to $1 / e$ of its peak value at zero separation.

One way for calculating correlation scales could be that described in Matthaeus et al. (1986) in which the original data set is divided in sub-intervals. The sole condition for including each subinterval in the averages is the degree to which it represents a weakly timestationary process. Statistical quantities as means, variances, correlation scales are obtained as ensemble averages. Effectively we get the correlation times from the autocorrelation functions. Frozen-in flow is used to convert correlation times to correlation lengths $\lambda_{C}$ using the mean solar wind speed in the subintervals. The typical values of correlation times we get, appear to be compatible with those reported for the magnetic field by various authors, including Matthaeus \& Goldstein (1982) and Bruno \& Dobrowolny (1986)

For this analysis, the magnetic fluctuations are computed using $2-3$ correlation times, and averaging the results over 2 days periods. We choose the reference position $r_{0}$ as the inner boundary of the observations, namely around 0.29 AU (Matthaeus et al. 1998, 2004). Table 2 summarizes the correlation times, the turbulence amplitudes $Z$ (computed from $81 \mathrm{~s}$ magnetic and velocity field data), cross helicity $\sigma_{c}$ (computed from 81 s magnetic and velocity field data), the factor $f$, the non-linear time $t_{N L}$ and the dimensionless age $\tau$ for each sample. 
In the simulation case, equation 5 becomes

$$
\tau(t)=\int_{t_{0}}^{t} \frac{d t^{\prime}}{t_{N L}\left(t^{\prime}\right)}
$$

where $t^{\prime}$ is the age and we do not need to know the spatial variation of the correlation scale and the turbulent energy density. In Figure 6 the turbulence amplitude $Z$ (in Alfven unit), the correlation length $\lambda_{C}$ (in box unit), the nonlinear time $t_{N L}$ (in $t$ unit) and the dimensionless age $\tau$ are shown as a function of the simulation time $t$. This is a decaying run, so the energy is low by the end of the run, and the turbulence amplitude $Z$ decreases about $40 \%$ of what it was initially.

The time history of the PVI fraction lying above $\theta$ for the Helios 2 data case, and the simulation data case, are displayed in Figure 7, for thresholds $\theta=3,4,5,6$. This plot shows a very strong similarity in the behavior of the PVI from Helios 2 and simulation data. In particular, the graphs corresponding to the same $\theta$ have the same trend. For example, those corresponding to "PVI>3" do not show large variations with $\tau$. On the contrary, the plots corresponding to "PVI $>6$ " display a rich behaviour in function of the dimensionless age. Clearly, we have much less points in the solar wind data set, but from the comparison with the simulation case, we can extrapolate the behaviour of the solar wind and say that the likelihood of finding coherent structures increases substantially up to $3-4$ nonlinear times,

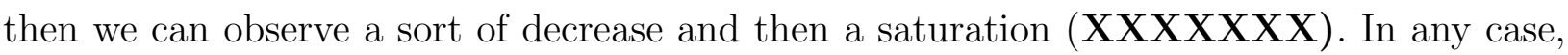
a clear development of intermittency is present as the turbulence ages.

\section{Discussion and Conclusions}

The purpose here has been to quantitatively evaluate the development of intermittency of the magnetic field fluctuations in the solar wind. In order to find a sensitive discriminator of this dynamical effect, we depart only slightly from usual intermittency measures. Integration of the cumulative probability of all values of the PVI statistic above a given threshold is a way to measure directly the population of vector increment values that are responsible for elevated values of higher order moments. Adopting this approach, we examined the associated weight $P(P V I>\theta)$ as a function of time, which provides a measure of the initial onset of intermittency in a simulation that begins from Gaussian fields containing no coherent structures. Here we have seen that this development of intermittency in simulation is very similar to that in $P(P V I>\theta)$ obtained from the analysis of Helios data in several streams over a range of heliocentric distances, and with varying solar wind speeds.

These results provide insight into how intermittency appears in solar wind fluctuations. First, the similarity of the initial increase and the subsequent variations in high PVI values 
in time in both solar wind and simulation data suggests that intermittency is formed in the solar wind through active in situ dynamics. Second, this observation provides still another indication that the solar wind is an example of such an active turbulent medium, and is not purely passive as has been sometime suggested. In particular, the present view is that the coherent structures are not passively advected but are themselves an integral part of the turbulent cascade. Finally, the similarity of the development of high PVI events in the solar wind and simulation offers the suggestion that the sources of the solar wind, perhaps near the Alfvenic critical region, may be incoherent. On the other hand, it would be difficult to imagine that the turbulence and structures seen in the lower corona are Gaussian and incoherent. Instead lower coronal turbulence appears to be highly intermittent (Dmitruk et al. 1998; Rappazzo et al. 2010). Therefore the present suggestion is that the way in which the solar wind is processed through the trans-Alfvenic region acts to destroy coherence, which then is dynamically built up again through nonlinear interactions in the super-Alfvenic wind. The latter point is more conjectural however and any such determination will await exploratory missions such as Solar Orbiter and Solar Probe.

This research supported in part by the NASA Heliophysics Theory Program (NNX08AI47G), the NSF SHINE program (ATM-0752135), and the Solar Probe ISIS Theory and Modeling project. A.G, S. S. and P. D. aknowledge the Marie Curie "Turboplasmas" project.

\section{REFERENCES}

Anselmet, F., Gagne, Y., Hopfinger, E. J., \& Antonia, R. A. 1984, J. Fluid Mech., 140, 63

Bavassano, B., Dobrowolny, M., Mariani, F., \& Ness , N. 1982, J. Geophys. Res., 87, 3617

Bavassano, B., \& Smith, E. J. 1986, J. Geophys. Res., 91, 1706

Belcher, J. W., \& Davis, L., Jr. 1971, J. Geophys. Res., 76, 3534

Biskamp, D. „\& Müller, W.-C. 2000, Phys. Plasmas, 7, 4889

Borovsky, J. E. 2008, J. Geophys. Res., 113, 8110

Breech, B., Matthaeus, W. H., Minnie, J., Oughton, S., Parhi, S., Bieber, J. W., \& Bavassano, B. 2005, Geophys. Res. Lett., 320, 6103

Breech, B., Matthaeus, W. H., Minnie, J., Bieber, J. W., Oughton, S., Smith, C. W., \& Isenberg, P. A 2008, J. Geophys. Res., 113, 8105 
Bruno, R., Bavassano, B., \& Villante, U. 1985, J. Geophys. Res., 90, 4373

Bruno, R., \& Dobrowolny, M. 1986, Ann. Geophys., 4, 17

Bruno, B., Carbone, V., Veltri, P., Pietropaolo, E., \& Bavassano 2001, B. Planet. Space Sci., 49,1201

Bruno, R., Carbone, V., Sorriso-Valvo, L., \& Bavassano, B. 2003, J. Geophys. Res.., 108, 8

Bruno, R., \& Carbone, V. 2005, Living Rev. Sol. Phys., 2, 4

Burlaga, L.F. 1991, J. Geophys. Res., 96, 5847

Carbone, V., Veltri, P., \& Mangeney, A. 1990, Phys. Fluids, 2, 1487

Carbone, V. 1994, Ann. Geophys., 12, 585

Carbone, V., Sorriso-Valvo, L., Lepreti, F., Veltri, P., \& Bruno, R. 2003, in AIP Conf. Proc., Solar Wind Ten, ed. M. Velli, R. Bruno, F. Malara, \& B. Bucci (Pisa, Italy), 679

Coleman, P. J., Jr. 1968, ApJ, 153, 371

D’Amicis, R., Bruno, R., Pallocchia, G., Bavassano, B., Telloni, D., Carbone, V., \& Balogh, A. 2010, ApJ, 717, 474

Dmitruk, P., Gómez, D. O., \& Deluca, E. E. 1998,ApJ505, 974

Dobrowolny, M., Mangeney, A., \& Veltri, P. 1980, Phys. Rev. Lett., 45, 144

Horbury, T. S., Balogh, A., Forsyth, R. J., \& Smith, E. J. 1997, Adv. Space Res., 19, 847

Frisch, U., Pouquet, A., Sulem, P.-L., \& Meneguzzi, M. 1983, Journal de Mecanique Theorique et Appliquee Supplement, 191

Greco, A., Chuychai, P., Matthaeus, W. H., Servidio, S., \& Dmitruk, P. 2008, Geophys. Res. Lett., 35, L19111

Greco, A., Matthaeus, W. H., Servidio, S., Chuychai, P., \& Dmitruk, P. 2009a, ApJ691, L111

Greco, A., Matthaeus, W. H., Servidio, S., \& Dmitruk, P. 2009b, Phys. Rev. E, 80, 046401

Horbury, T. S., \& Balogh, A. 1997, Nonlin Processes Geophys., 4, 185

Kasper, J. C., Lazarus, A. J., \& Gary, S. P. 2008, Phys. Rev. Lett., 101, 261103 
Klein, L. W., Matthaeus, W. H., Roberts, D. A., \& Goldstein, M. L. 1992, in AIP Conf. Proc., Solar Wind Seven, ed. E. Marsch \& R. Schwenn (Goslar, Germany)

Marsch, E., \& Tu, C. Y. 1990, J. Geophys. Res., 95, 8211

Marsch, E., \& Tu, C. Y. 1994, Ann. Geophys., 12, 1127

Matthaeus, W. H., \& Montgomery, D. 1980, Annals of the New York Academy of Sciences, 357,203

Matthaeus, W. H., \& Goldstein, M. L. 1982, J. Geophys. Res., 87, 6011

Matthaeus, W. H., \& Goldstein, M. L. 1986, Phys. Rev. Lett., 57, 495

Matthaeus, W. H., Goldstein, M. L., \& King, J. H. 1986, J. Geophys. Res., 91, 59

Matthaeus, W. H., Smith, C. W., \& Oughton, S. 1998, J. Geophys. Res., 103, 6495

Matthaeus, W. H., Minnie, J., \& Breech, B. 2004, Geophys. Res. Lett., 311, 12803

Matthaeus, W. H., Dasso, S., Weygand, J. M., Milano, L. J., Smith, C. W., \& Kivelson, M. G. 2005, Phys. Rev. Lett., 95, 231101.

Ng, C. S., Bhattacharjee, A., Munsi, D., Isenberg, P. A., \& Smith, C. W. 2010, J. Geophys. Res., 115, 2101

Rappazzo, A. F., Velli, M., \& Einaudi, G. 2010, ApJ, 722, 65

Roberts, D. A., Goldstein, M. L., Klein, L. W., \& Matthaeus, W. H. 1987, J. Geophys. Res., 921,12023

Roberts, D. A., Goldstein, M. L., Matthaeus, W. H., \& Ghosh, S. 1992, J. Geophys. Res., 971,17115

Servidio, S., Matthaeus, W. H., Shay, M. A., Dmitruk, P., Cassak, P. A., \& Wan, M. 2010, Phys. Plasmas 17, 032315

Smith, C. W., Matthaeus, W. H., Zank, G. P., Ness, N. F., Oughton, S., \& Richardson, J. D. 2001, J. Geophys. Res., 106, 8253

Sorris-Valvo, L., Carbone, V., \& Veltri, P. 1999, Geophys. Res. Lett., 26, 1801

Sreenivasan, K. R., \& Antonia, R. A. 1997, Annual Review of Fluid Mechanics, 29, 435

Tsurutani, B. T., \& Smith, E. J. 1979, J. Geophys. Res., 84, 2773. 
Tu, C. Y., Pu, Z.Y., \& Wei, F. S. 1984, J. Geophys. Res., 89, 9695

Tu, C. Y. 1988, J. Geophys. Res., 93, 720

Tu, C. Y., Marsch, E., \& Thieme, K. M. 1989, J. Geophys. Res., 94, 11739

Tu, C. Y., \& Marsch, E. 1993, J. Geophys. Res., 98, 1257

Tu, C. Y., \& Marsch, E. 1995, Space Sci. Rev., 73, 1

Tu, C. Y., Marsch, E., \& Rosenbauer, H. 1996, Ann. Geophysicae, 14, 270

Veltri, P., \& Mangeney, A. 1999, in AIP Conf. Proc., Solar Wind IX, ed. S. Habbal, J. V. Hollweg, P. A. Isenberg, \& R. Esser (Nantucket, MA, USA), 471

Wan, M., Oughton, S., Servidio, S., \& Matthaeus, W. H. 2009, Phys. Plasmas, 16, 080703

Zank, G. P., Matthaeus, W. H., \& Smith, C. W. 1996, J. Geophys. Res., 1011, 17093 


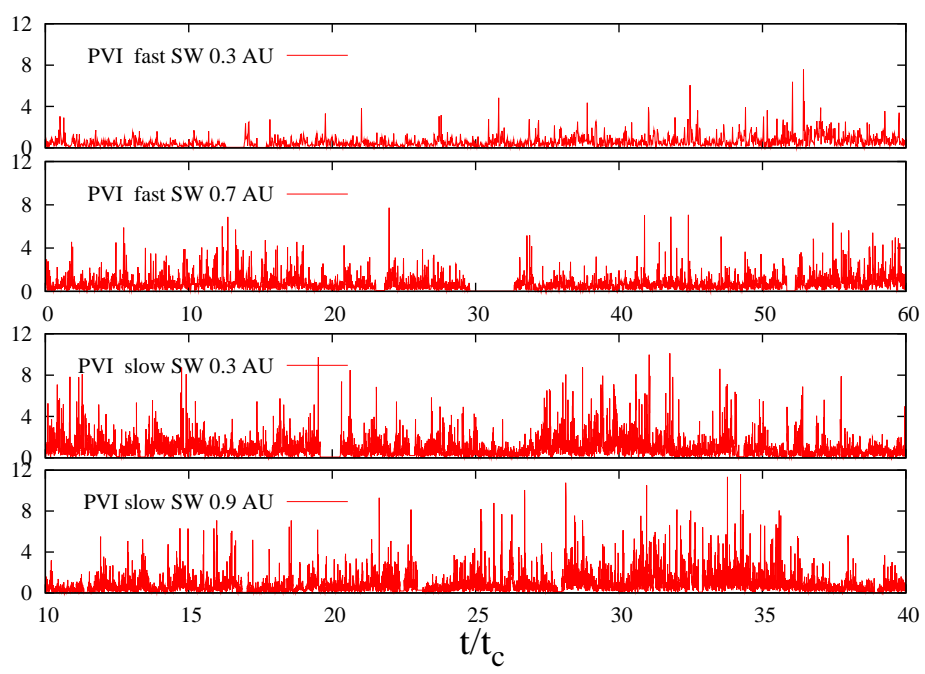

Fig. 1. - Time series of PVI computed for some of the six streams from solar wind. Time is normalized to the correlation time. Here we show only a fraction of the whole time interval.

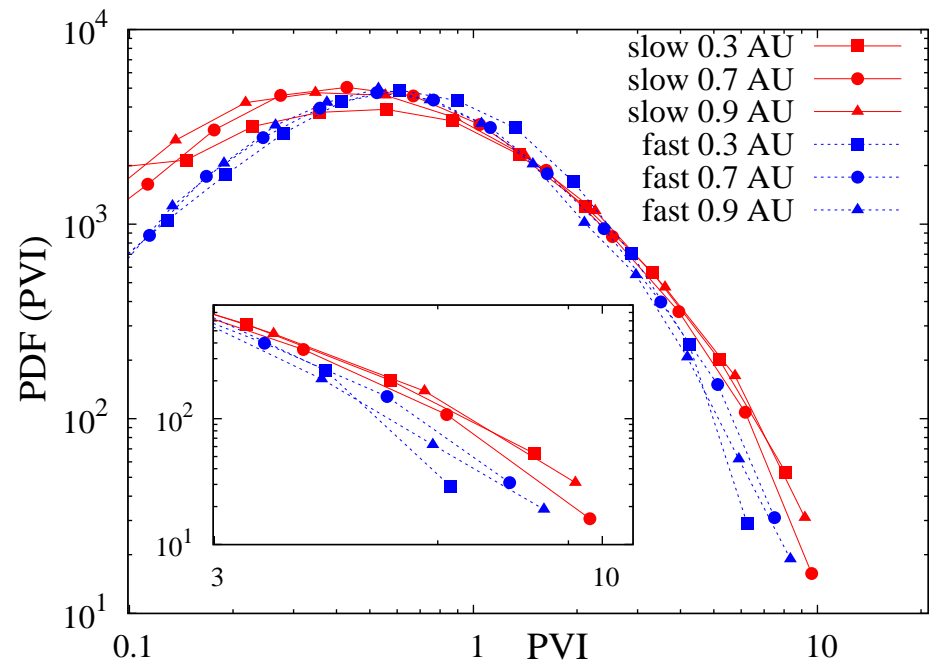

Fig. 2.- PDFs of PVI magnitude for all the six streams in solar wind. Red lines refer to slow wind streams and blue lines to fast slow streams. The inset show sa blow-up in the range of PVI values larger than 3, in order to better examine the tails of the distributions. The error bars are of the same order of the point thickness. (A color version of this figure is available in the online journal). 


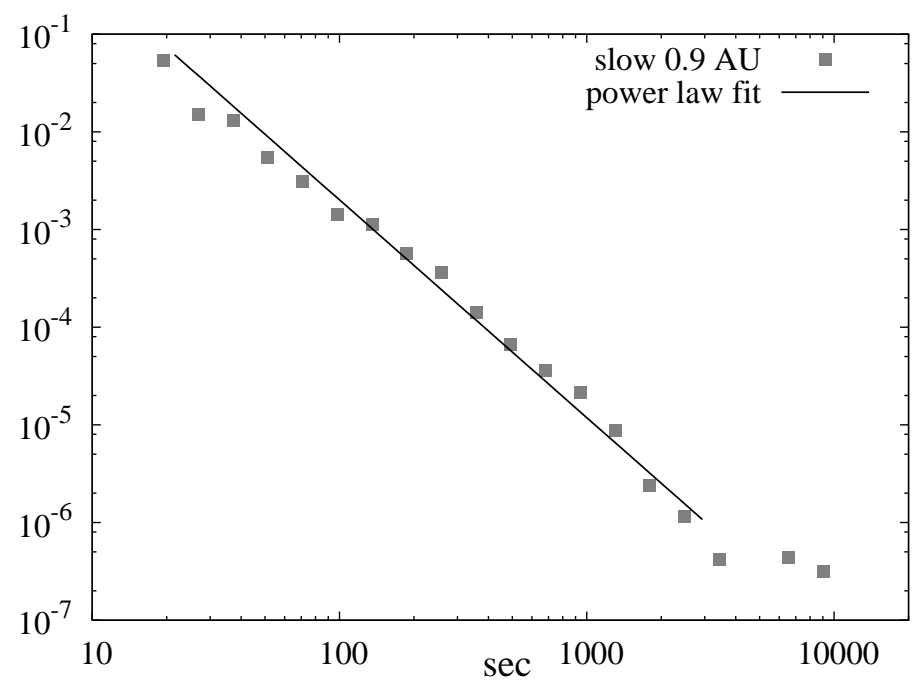

Fig. 3.- Distributions of waiting times between discontinuities detected in slow solar wind (squares) by Helios 2 at $R=0.9 \mathrm{AU}$. The line is the power law fit $a x^{-b}$. The value of the scaling exponent is $b=2.3 \pm 0.1$.

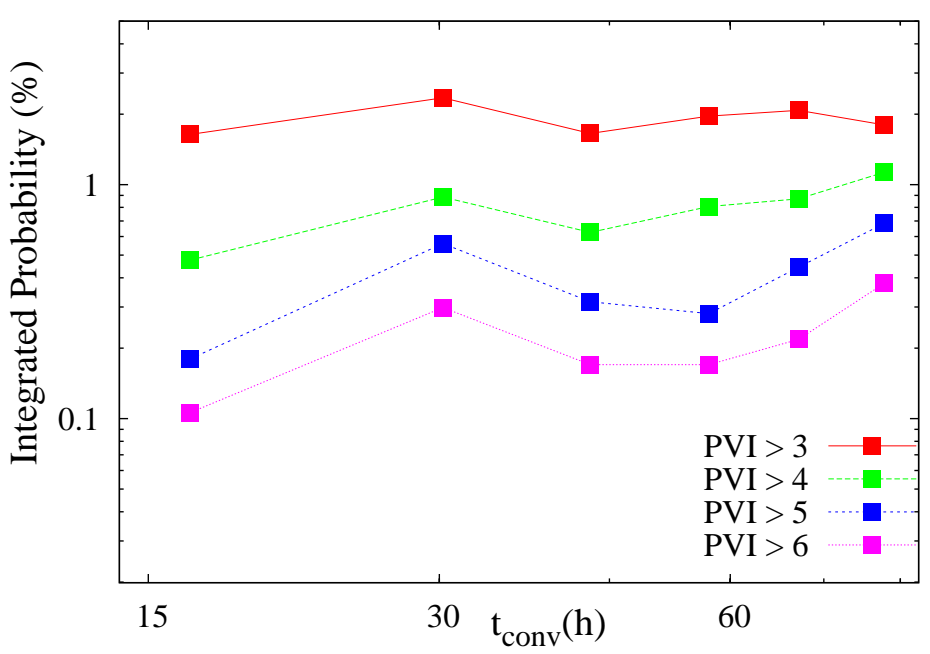

Fig. 4.- PVI distribution functions integrated over all values $>\theta=$ 3 (red), 4 (green), 5 (blue), 6 (magenta) vs the convective age of solar wind. The error bars are of the same order of the point thickness. (A color version of this figure is available in the online journal). 


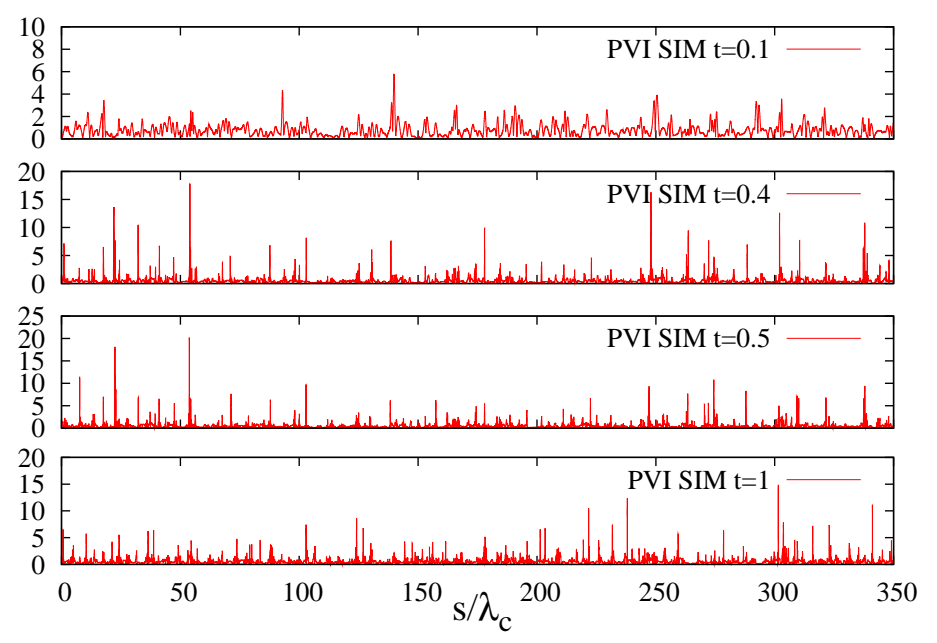

Fig. 5.- PVI series computed for 4 instants from the 2D simulation. Space is normalized to the correlation scale $\lambda_{C}=1.8 \times 10^{-1}$.
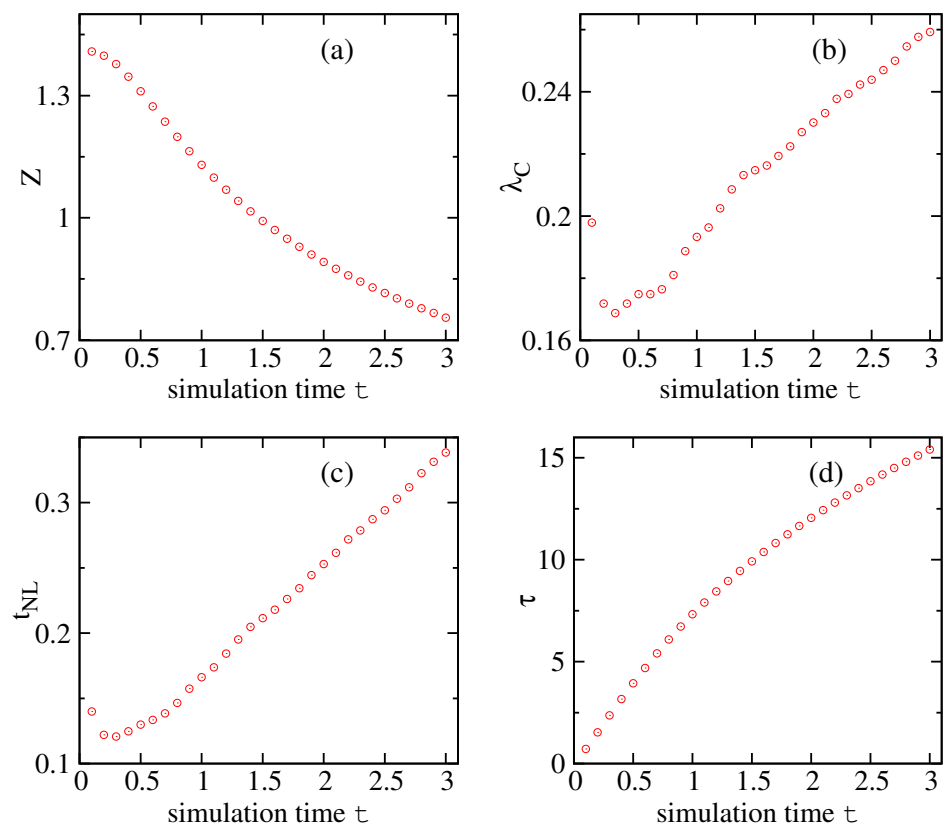

Fig. 6. - Time history of the turbulence amplitude $Z$ (panel (a)), the the correlation length $\lambda_{C}$ (panel (b)), the nonlinear time $t_{N L}$ (panel (c)) and the dimensionless age $\tau$ (panel (d)) are shown in function of the simulation time $t$. 

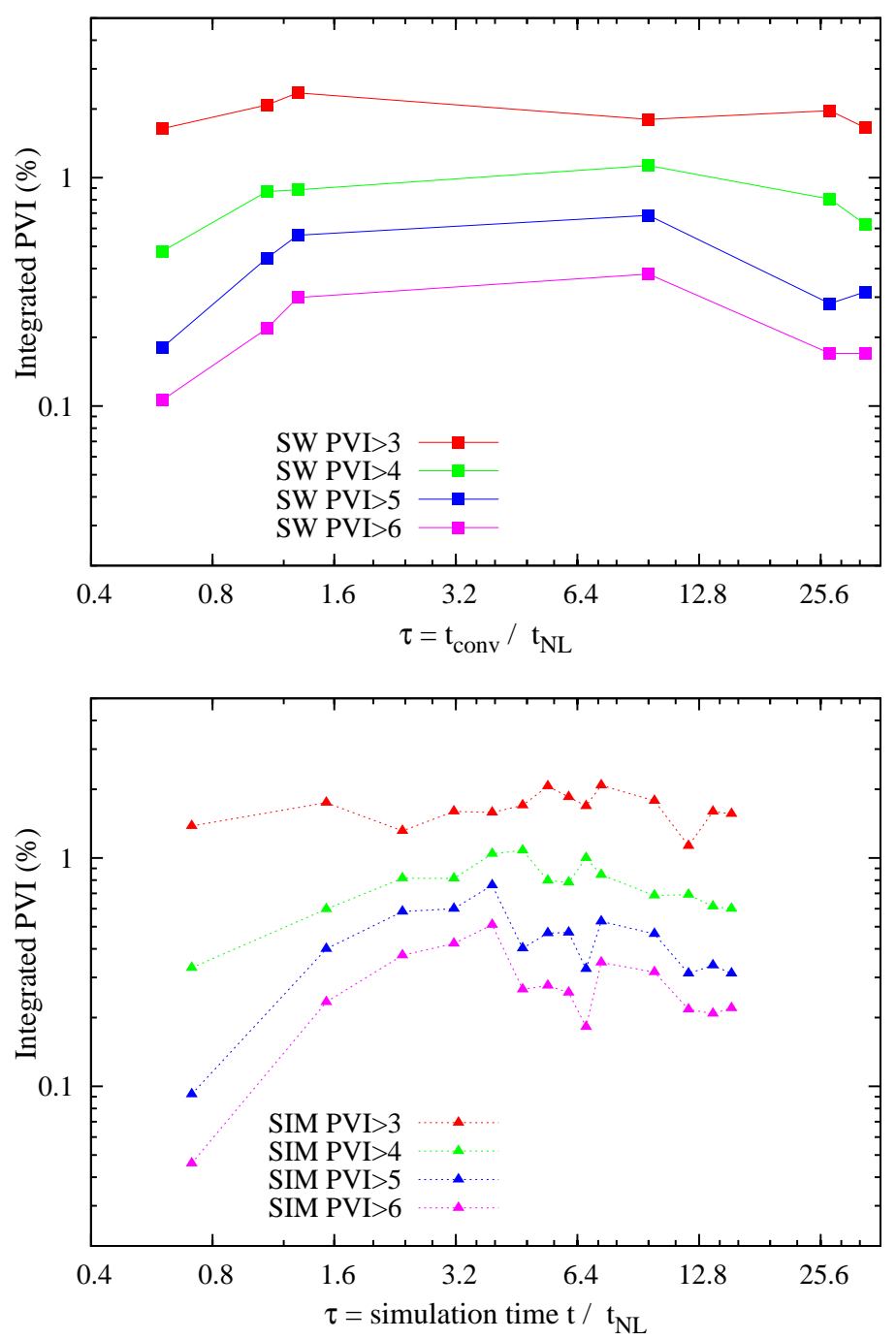

Fig. 7.- Time history of the PVI distribution functions integrated over all values $>$ $\theta=3$ (red), 4 (green), 5 (blue), 6 (magenta). Simulation (triangles) and solar wind (squares) times are normalized to the corresponding nonlinear times. (A color version of this figure is available in the online journal). 
Table 1: Selected time intervals for Helios 2. Time interval, heliocentric distance $R$, average solar wind speed $U$, the solar wind convective age $t_{c o n v}$ and number of analyzed data points are shown from left to right.

\begin{tabular}{ccccc}
\hline $\begin{array}{c}\text { time interval } \\
\text { [ddd:hh - ddd:hh] }]\end{array}$ & $\begin{array}{c}R \\
{[\mathrm{AU}]}\end{array}$ & $\begin{array}{c}U \\
{[\mathrm{~km} / \mathrm{s}]}\end{array}$ & $\begin{array}{c}t_{\text {conv }} \\
{[\mathrm{h}]}\end{array}$ & No. of points \\
\hline 49:14- $51: 14$ & 0.88 & 643 & 57 & 28800 \\
$75: 12-77: 12$ & 0.65 & 630 & 43 & 28800 \\
105:12- 107:12 & 0.29 & 729 & 16.6 & 28800 \\
& & & & \\
46:00- 48:00 & 0.90 & 433 & 86.6 & 28794 \\
$72: 00-74: 00$ & 0.69 & 412 & 70.8 & 27943 \\
$99: 12-101: 12$ & 0.34 & 405 & 30.9 & 28800 \\
& & & & \\
\hline
\end{tabular}


Table 2: The solar wind convective age $t_{c o n v}$, correlation times, turbulence amplitudes $Z$, cross helicity $\sigma_{c}, f$, the non-linear times $t_{N L}$, and the dimensionless ages $\tau$ of analyzed data set are shown from left to right.

\begin{tabular}{ccccccr}
\hline $\begin{array}{c}t_{\text {conv }} \\
{[h]}\end{array}$ & $\begin{array}{c}\text { correlation time } \\
{[\mathrm{s}]}\end{array}$ & $\begin{array}{c}Z \\
{[\mathrm{~km} / \mathrm{s}]}\end{array}$ & $\sigma_{c}$ & $f$ & $\begin{array}{c}t_{N L} \\
{[\mathrm{~s}]}\end{array}$ & $\tau$ \\
\hline & & & & & & \\
57 & 780 & 36 & 0.76 & 0.6 & 8346 & 27 \\
43 & 440 & 38 & 0.82 & 0.51 & 3708 & 33 \\
16.6 & 205 & 72 & 0.84 & 0.48 & 996 & 0.6 \\
& & & & & & \\
86.6 & 1960 & 23 & 0.2 & 0.97 & 35875 & 9.6 \\
70.8 & 15990 & 30 & 0.42 & 0.885 & 197157 & 1.09 \\
30.9 & 1515 & 36 & 0.37 & 0.9 & 15377 & 1.3 \\
& & & & & & \\
\hline
\end{tabular}

